\title{
A Saturated-Interface-Volume Phase Change Model for Simulating Flow Boiling ${ }^{\S}$
}

\author{
Zhenhai Pan, Justin A. Weibel, Suresh V. Garimella" \\ Cooling Technologies Research Center, an NSF I/UCRC \\ School of Mechanical Engineering \\ Purdue University \\ West Lafayette, IN 47907 USA
}

\begin{abstract}
High-fidelity simulation of flow boiling in microchannels remains a challenging problem, but the increasing interest in applications of microscale two-phase transport highlights its importance. In this paper, a volume of fluid (VOF)-based flow boiling model is proposed with features that enable cost-effective simulation of two-phase flow and heat transfer in realistic geometries. The vapor and liquid phases are distinguished using a color function which represents the local volume fraction of the tracked phase. Mass conservation is satisfied by solving the transport equations for both phases with a finite-volume approach. In order to predict phase change at the liquid-vapor interface, evaporative heat and mass source terms are calculated using a novel, saturated-interface-volume phase change model. This phase change model is formulated to anchor the interfacial temperature at saturation within each iteration, and thereby acts as a robust constant-temperature boundary condition. Unlike other available phase-change models, the source terms are coupled with the local temperature explicitly; therefore, numerical oscillations around the interface temperature are not observed during iterations within a time step, which reduces the numerical cost. In addition, the reference frame is set to move with the vapor slug to artificially increase the local velocity magnitude in the thin liquid film region in the relative frame. This reduces the influence of numerical errors resulting from calculation of the surface tension force, and thus suppresses the development of spurious currents. As a result, non-uniform meshes may be used which can efficiently resolve high-aspect-ratio geometries and flow features. The overall numerical expense is significantly reduced. The proposed saturated-interface-volume model is first validated against a one-dimensional Stefan problem, and then used to simulate the growth of a vapor bubble flowing in a heated, 2D axisymmetric microchannel. The bubble motion, bubble growth rate, liquid film thickness, and local heat transfer coefficient along the wall are compared against previous numerical studies. A three-dimensional flow boiling problem is studied to demonstrate the cost effectiveness of the present approach and to highlight the transport mechanisms it can reveal in more complex domains.
\end{abstract}

\footnotetext{
${ }^{\S}$ Submitted for possible publication in International Journal of Heat and Mass Transfer, August 2015.

" Author to whom correspondence should be addressed: sureshg@purdue.edu
} 
Keywords: flow boiling; microchannel; two phase; phase change; VOF simulation

\section{INTRODUCTION}

Increasing recent attention to microchannel flow boiling has resulted from the demand for highperformance, compact cooling systems. The latent heat absorbed during vaporization confers flow boiling with higher heat transfer coefficients and more uniform surface temperatures than single-phase convective heat transfer, which allows for operation at a lower mass flow rate and thereby a smaller pressure drop. Accurate numerical simulations can reveal details of the heat and mass transfer processes that are not measureable in experiments; this would improve the understanding of key transport mechanisms as well as predictions of both global and local heat transfer performance.

A number of numerical methods have been developed to study phase change [1][2]. The interface is evaluated either by a Lagrangian [3][4] or an Eulerian method [5][6][7][8]. In Lagrangian methods, the interface is represented by mesh faces, which allows for an accurate evaluation of the interface normal gradient [9][10]. However, it is expensive to track complex interfacial deformation in transient problems using a moving mesh with Lagrangian methods. In Eulerian methods, the interface geometry is reconstructed from a color function that is used to track the phases, such as the volume fraction in volume-of-fluid (VOF) methods. This allows convenient tracking of complex interface deformations [11][12].

Among the various numerical techniques available, VOF methods are promising for resolving phase change heat transfer due to the intrinsic mass conservation achieved, unlike with other Eulerian methods. However, fixed-grid approaches must estimate the interface profile; VOF methods may thereby induce unphysical flows (referred to as spurious currents) due to the numerical error in estimating the interfacial surface tension. Numerous approaches have been proposed to suppress spurious currents: Gupta et al. [13] suggested a square mesh and a Green-Gauss node-based method for calculating pressure gradients; Magnini et al. [14][15] coupled VOF with a height-function method [16] using a uniform mesh; and Sussman and Puckett [17]and Sun and Tao [18]coupled VOF with a level-set (LS) method to improve the interface profile accuracy. However, the underlying need for uniform square mesh cells in these approaches has high computational cost. Most recently, Pan et al. [19] demonstrated that spurious currents can be suppressed by increasing the local velocity near the interface by employing an artificial moving reference frame. This specific treatment uniquely allows the use of 
high-aspect-ratio mesh elements, and thus can significantly reduce the computational expense when simulating high-aspect-ratio physical features, such as thin liquid films and rectangular microchannels.

Formulating the interface phase change model is another primary challenge in the modeling of flow boiling, and typically results in an inevitable tradeoff between physical accuracy and computational cost. A simplifying saturated model is commonly employed [20] where the evaporative mass source is defined as

$$
S_{m}=r C_{l} \rho_{l} \frac{\left(T-T_{s a t}\right)}{T_{s a t}},
$$

where $r$ is an empirical coefficient used to bring the temperature of the cells containing the interface close to saturation, and can range from $0.1 \mathrm{~s}^{-1}$ to $100 \mathrm{~s}^{-1}$ [21][22][23]. In general, an overly large or small value of $r$ shifts the interfacial temperature away from the saturation temperature; a large value of $r$ may also induce numerical instabilities that cause the interfacial temperature to oscillate around the saturation temperature. Later improvements to the saturated phase change model [24][25][26][27][28] introduced physics-based expressions that avoid empiricism. The phase change mass source is calculated based on the local heat flux on the interface as

$$
S_{m}=\left(\left.k_{l} \frac{\partial T}{\partial \vec{n}_{l}}\right|_{\text {int }}+\left.k_{v} \frac{\partial T}{\partial \vec{n}_{v}}\right|_{\text {int }}\right) \frac{A_{\text {int,cell }}}{V_{\text {cell }}},
$$

where $\vec{n}_{i}$ is the unit normal vector pointing to the $i^{\text {th }}$ phase and $A_{\text {int,cell }}$ is the interface area in the cell. The temperature gradient on the interface $\partial T / \partial \vec{n}_{i}$ can be calculated as $\partial T / \partial \vec{n}_{i}=\left(T_{\text {cell }}-T_{\text {sat }}\right) / d$ where $d$ is the distance from the cell center to the interface [25], or simplified as the temperature gradient in the cell $\nabla T_{\text {cell }}$ [26][28].

During the evaporation process, the interfacial temperature exceeds the saturation temperature to overcome the interfacial evaporative resistance. This interfacial resistance is typically much smaller than the thermal resistance of the adjacent liquid phase, and this temperature rise is usually only important in three-phasecontact-line regions with a submicron-scale liquid film [29]. The interfacial temperature rise above saturation can be evaluated as [30]

$$
m^{\prime \prime}=\phi\left(T-T_{\text {sat }}\right), \phi=\frac{2 \gamma}{2-\gamma}\left(\frac{M}{2 \pi R}\right)^{1 / 2}\left(\frac{\rho_{v} h_{f g}}{T_{s a t}^{3 / 2}}\right)
$$

By coupling Eq. (2) and Eq. (3), Kunkelmann and Stephan [31] and Magnini et al. [14][15] investigated boiling with a non-equilibrium phase change model that includes the temperature rise above the saturation temperature at an evaporating interface. Both the equilibirum and non-equilibrium phase change models suffer from 
numerical instabilities and must rely upon the source-term redistribution scheme proposed by Hardt and Wondra [32], in which the mass sources are artificially diffused across several cell layers adjacent to the interface.

The above phase change models aim to obtain an interface temperature (either saturated or unsaturated) by iteratively solving the governing transport equations, which requires multiple iterations within a time step. For microscale flow boiling problems, the liquid film between the superheated wall and the liquid-vapor interface determines the heat transfer coefficient and thus must be accurately captured. Combined with the need for uniform square mesh cells to suppress spurious currents, the computational cost can be prohibitive. In the current study, we develop a numerical modeling approach to simulate the growth of a vapor slug traveling through a heated microchannel. Although such problems have been widely studied in the literature, the costeffective modeling approach presented in this paper can be easily implemented with basic VOF solvers. Spurious currents are suppressed by introducing a moving reference frame that allows the adoption of a nonuniform mesh setup with high-aspect-ratio cells. A novel, saturated-interface-volume phase change model is proposed to account for the heat and mass exchange at the interface, and for which the interface is fixed at the saturation temperature for each iteration. Iteration of the interface temperature is not required for simulating the phase change process, thus significantly reducing the computational cost without sacrifice of physical accuracy relative to other techniques. After validation of the phase-change model, flow boiling predictions are quantitatively compared against past approaches. The growth of a vapor slug traveling in a long, square, heated channel is then investigated to demonstrate the utility of the present model.

\section{NOMENCLATURE}

\begin{tabular}{|c|c|}
\hline$A$ & area $\left(\mathrm{m}^{2}\right)$ \\
\hline$C$ & volume fraction \\
\hline$c_{p}$ & heat capacity $(\mathrm{J} / \mathrm{kg} \mathrm{K})$ \\
\hline$D$ & channel diameter $(\mathrm{m})$ \\
\hline$F$ & volumetric body force $\left(\mathrm{N} / \mathrm{m}^{3}\right)$ \\
\hline$h_{f g}$ & latent heat $(\mathrm{J} / \mathrm{kg})$ \\
\hline$K$ & thermal conductivity $(\mathrm{W} / \mathrm{m} \mathrm{K})$ \\
\hline$L$ & channel length (m) \\
\hline$M$ & molecular weight $(\mathrm{kg} / \mathrm{mol})$ \\
\hline$m^{\prime \prime}$ & mass flux $\left(\mathrm{kg} / \mathrm{m}^{2} \mathrm{~s}\right)$ \\
\hline$\vec{n}$ & unit normal vector \\
\hline
\end{tabular}




$\begin{array}{ll}P & \text { pressure }\left(\mathrm{N} / \mathrm{m}^{2}\right) \\ q^{\prime \prime} & \text { heat flux }\left(\mathrm{W} / \mathrm{m}^{2}\right) \\ R & \text { universal gas constant }(\mathrm{J} / \mathrm{mol} \mathrm{K}) \\ S_{h} & \text { energy source }\left(\mathrm{W} / \mathrm{m}^{3}\right) \\ S_{m} & \text { mass source }\left(\mathrm{kg} / \mathrm{m}^{3} \mathrm{~s}\right) \\ T & \text { temperature }(\mathrm{K}) \\ T & \text { time }(\mathrm{s}) \\ \Delta t & \text { time step }(\mathrm{s}) \\ U & \text { velocity magnitude }(\mathrm{m} / \mathrm{s}) \\ \vec{U} & \text { velocity vector }(\mathrm{m} / \mathrm{s}) \\ \Delta t & \text { time step (s) } \\ W & \text { width (m) } \\ X & \text { axial location }(\mathrm{m}) \\ \Delta x & \text { mesh size }(\mathrm{m}) \\ Y & \text { radial location }(\mathrm{m}) \\ Z & \text { flow direction location }(\mathrm{m})\end{array}$

\section{Greek}

$\begin{array}{ll}\Delta & \text { film thickness }(\mathrm{m}) \\ \delta_{s} & \text { delta function } \\ \Gamma & \text { accommodation coefficient } \\ M & \text { dynamic viscosity }\left(\mathrm{N} \mathrm{s} / \mathrm{m}^{2}\right) \\ K & \text { interface curvature }\left(\mathrm{m}^{-1}\right) \\ P & \text { density }\left(\mathrm{kg} / \mathrm{m}^{3}\right) \\ \Sigma & \text { surface tension }(\mathrm{N} / \mathrm{m})\end{array}$

\section{Subscript}

$\begin{array}{ll}\text { O } & \text { static reference } \\ A x & \text { axial component } \\ C & \text { critical } \\ \text { Cell } & \text { center value of the cell } \\ I & \text { the } \text { i }^{\text {th }} \text { phase } \\ \text { In } & \text { initial } \\ \text { Int } & \text { interface } \\ L & \text { liquid } \\ \text { La } & \text { latent } \\ N & \text { time step }\end{array}$




$\begin{array}{ll}R & \text { relative } \\ \text { Ra } & \text { radial component } \\ \text { Ref } & \text { reference frame } \\ S & \text { surface tension } \\ \text { Sat } & \text { saturated } \\ \text { Tar } & \text { target } \\ V & \text { vapor } \\ W & \text { wall }\end{array}$

\section{NUMERICAL MODEL}

\subsection{Volume of Fluid (VOF) Implementation}

The volume of fluid method is an Eulerian approach in which the mesh is fixed and does not move with the interface. The two different phases are separated based on a so-called color function $C$, which represents the local volume fraction of the tracked phase: $C=1$ in cells filled with the tracked phase and $C=0$ in cells filled with the other phase. Cells with $0<C<1$ are recognized as containing the interface. The motion of the interface is captured by solving the continuity equation

$$
\frac{\partial\left(C_{i} \rho_{i}\right)}{\partial t}+\nabla \cdot\left(C_{i} \rho_{i} \vec{U}\right)=S_{m, i}
$$

In the bulk, Eq. (4) becomes

$$
\frac{\partial \rho}{\partial t}+\nabla(\rho \vec{U})=S_{m, i}
$$

With this treatment, the mass of the two phases is conserved and the two-phase flow problem can be solved with a single-phase formulation. For Newtonian fluids in laminar flow, the momentum and energy equations are written as

$$
\begin{gathered}
\frac{\partial(\rho \vec{U})}{\partial t}+\nabla(\rho \vec{U} \cdot \vec{U})=-\nabla p+\nabla\left(\mu\left(\nabla \vec{U}+\nabla \vec{U}^{T}\right)\right)+F_{s} \\
\frac{\partial\left(\rho c_{p} T\right)}{\partial t}+\nabla\left(\rho c_{p} T \vec{U}\right)=\nabla \cdot(\mathrm{k} \nabla T)+S_{h}
\end{gathered}
$$

The fluid properties are determined from the volume-fraction-weighted average of the properties of the two phases. The surface tension force $F_{s}$, based on the continuum surface force (CSF) model [33], is treated as a 
volumetric body force, and is implemented in the cells around the interface where the gradient of volume fraction $\nabla C \nabla C$ is not equal to zero.

$$
F_{s}=\sigma \kappa \delta_{s} \vec{n}=-\sigma \nabla \cdot\left(\frac{\nabla C}{|\nabla C|}\right)|\nabla C|
$$

With the above treatment, the surface tension force should be calculated to be normal to the interface using an accurate value of $\nabla C$; however, since the volume fraction $C$ in the interface region is not a continuous field, but is instead discretized over the mesh, it is difficult to obtain an exact $\nabla C$ from VOF simulations; errors in the surface tension force may produce unphysical spurious velocities. In the present study, we suppress spurious currents by employing the moving reference frame method [19], as described in Section 4.

\subsection{Saturated-Interface-Volume Phase Change Model}

The mass source $S_{m}$ in the continuity equation and the energy source $S_{h}$ in the energy equation account for phase change at the interface. State-of-the-art phase change models may be classified either as equilibrium models where the interfacial temperature is assumed to be saturated or as non-equilibrium models where there is an interfacial temperature rise above the saturated temperature to maintain evaporation. While the nonequilibrium model provides a more accurate prediction of the evaporation rate, the equilibrium model is sufficiently valid up to a maximum heat flux that is larger than typically encountered values, and is preferred for simplicity. The interfacial temperature rise above saturation during the evaporation process may be evaluated using Eq. (3). For non-polar liquids, the accommodation coefficient $\gamma$ is equal to 1. For example, for R113 ( $\Phi \sim$ $\left.23.7 \mathrm{~kg} / \mathrm{m}^{2} \mathrm{sK}\right)$ the interfacial temperature rise above saturation is less than $0.1 \mathrm{~K}$ for local evaporative heat fluxes at the interface of up to $170 \mathrm{~kW} / \mathrm{m}^{2}$. Under the operating conditions investigated in the present study, the maximum temperature jump is thus smaller than $0.01 \mathrm{~K}$, and is negligible.

For an equilibrium phase change model, the latent heat of vaporization must maintain a saturated interface temperature and keep the vapor phase saturated. Assuming that the entire volume of the cell containing the interface is saturated (as illustrated in Fig. 1(a)), the saturated-interface-volume phase change model would yield the energy source term for the latent heat in the cells where $0<C_{v}<1$ as

$$
S_{h, l a}=\frac{C_{l} \rho_{l} c_{p l}\left(T_{t a r}-T\right)+C_{v} \rho_{v} c_{v l}\left(T_{t a r}-T\right)}{\Delta t}
$$

where $T$ is evaluated at the cell centroid before the source term is added and $T_{\text {tar }}$ is the target temperature that the

cell centroid should be fixed to the energy source is added. In the saturated-interface-volume phase change model, the target temperature $T_{t a r}$ is equal to the saturation temperature. This phase change model makes the 
same physical assumptions about the evaporation process as prior equilibrium modeling approaches $[25][27][28]$. However, as in the non-equilibrium models [14][15][31], it assumes that the interfacial temperature is represented by the central temperature of the cells containing the interface (Fig. 1(a)). The source terms are thereby simply determined based on an energy balance over the entire cell volume containing the interface (i.e., temperature gradient between cells) to maintain a saturated interface, rather than attempting to calculate a temperature gradient normal to the interface within the cell. The vapor and liquid mass source is then calculated as

$$
S_{m, v}=-S_{m, l}=-S_{h, l a} / h_{f g}
$$

Wherever a mass source is added, a corresponding sensible energy source should be added in the same cell, calculated as

$$
S_{h, s}=S_{m, l} c_{p l}\left(T-T_{\text {reference }}\right)+S_{m, \mathrm{v}} c_{p v}\left(T-T_{\text {reference }}\right)
$$

In the commercial software ANSYS FLUENT 15.0 [34] employed in the present simulations, the reference temperature $T_{\text {reference }}$ is $298.15 \mathrm{~K}$.

The flow chart of the saturated-interface-volume phase change model at one time step is shown in Fig. 1(b); the steps are as follows:

1. The location of the interface is updated after the continuity and momentum equations converge.

2. Cells containing the interface $\left(0<C_{v}<1\right)$ are marked.

3. Conductive and convective heat transfer rates into or out of all cells (including the interfacial cells) are calculated based on the local velocity and temperature field.

4. The temperature field is updated and the temperature of the interfacial cells is shifted away from the saturation temperature.

5. The latent energy source is added using the explicit formation provided in Eq. (9) to force the temperature of the interfacial cells to return exactly to the saturation temperature.

6. Steps 3-5 are repeated until the temperature field is converged. Note that Step 5 fixes the initial interfacial temperature for Step 3 at saturation throughout the iteration process, which acts like a constant-temperature boundary condition on the interface, and thus accelerates convergence.

The primary advantage of the phase change model developed here is that the interface is fixed at the saturation temperature at each iteration using Eq. (9), as illustrated in Fig. 1(b). The process of setting the temperature at the interface to the saturation value does not require any nested iteration; numerical oscillations are not observed within a time step. 
One potential unphysical mechanism encountered in several instances is caused by the vapor mass source in Eq. (10), when a trailing interface moves out of a cell near the heated wall. The vapor mass fraction should decrease to zero when the interface moves out of the cell; however, the temperature is fixed at saturation and mass continues to be added to the cell. As a result, the volume fraction does not reduce to zero when the local evaporation flux is high; pinning of the interface at these cells causes the interface to be unstable and fragmented. To remedy this problem (as illustrated in Fig. 1(a)), the vapor mass sources (and their corresponding sensible energy) need to be moved to the nearest mesh cell with $C_{v}=1$. This correction need be applied only to those cells that have a vapor fraction lower than a selected critical value $C_{v, c}$ (as opposed to other source term redistribution approaches [32] that are imposed universally). The higher the choice of $C_{v, c}$, the more readily is this unphysical phenomenon avoided, but more interfacial mass is redistributed. In the present study, $C_{v, c}$ is conservatively set to 0.2 for the $2 \mathrm{D}$ axisymmetric case (Section 3.1 ) and 0.4 for the $3 \mathrm{D}$ case (Section 3.2 ) to ensure numerical stability while reducing the need to redistribute source terms on a corresponding percentage of the interfacial cells.

\section{PROBLEM DESCRIPTION}

We first simulate two-dimensional slug flow boiling (as illustrated in Fig. 2(a)) where a single vapor bubble travels into a heated microchannel to allow a direct comparison with a case considered in the literature [14]. We then investigate a similar flow boiling problem in a three-dimensional square microchannel.

\subsection{D Axisymmetric Flow Boiling in a Circular Microchannel}

The two-dimensional flow boiling case is shown in Fig. 2(b). The channel (of diameter $D=0.5 \mathrm{~mm}$ ) is divided into an $8 D$-long upstream adiabatic section and a $14 D$-long downstream heated length with a uniform heat flux of $9 \mathrm{~kW} / \mathrm{m}^{2}$ applied to the wall. Saturated liquid R113 enters the channel inlet with a uniform velocity of $0.4 \mathrm{~m} / \mathrm{s}$ (corresponding to a mass flux of $600 \mathrm{~kg} / \mathrm{m}^{2} \mathrm{~s}$ ) and temperature of $323.15 \mathrm{~K}$. The steady, single-phase temperature and flow fields are first solved to initialize the conditions for study of the flow boiling problem. After the single-phase initialization, a vapor bubble is defined in the adiabatic section with spherical cap shapes on both ends at time $t=0$; the initial bubble length is $3 D$ and its initial diameter is $0.92 D$.

\subsection{D Flow Boiling in a Square Microchannel}

In the three-dimensional problem, the channel (of width $W=0.5 \mathrm{~mm}$ ) is divided into a $6 W$-long upstream adiabatic section and a $10 \mathrm{~W}$-long downstream heated length with a uniform heat flux of $30 \mathrm{~kW} / \mathrm{m}^{2}$ applied to the 
wall. Saturated liquid R113 enters the channel inlet with a fully-developed velocity profile (corresponding to an average velocity of $0.6 \mathrm{~m} / \mathrm{s}$ ) and temperature of $323.15 \mathrm{~K}$. The steady, single-phase temperature and flow fields are first solved to initialize the conditions for study of the flow boiling problem. At time $t=0$, a vapor bubble is initialized in the adiabatic section with spherical cap shapes on both ends; the initial bubble length is $2.8 \mathrm{~W}$ and its initial diameter is $0.8 \mathrm{~W}$.

\section{NUMERICAL IMPLEMENTATION}

The numerical solution is obtained using the pressure-based finite volume scheme implemented in the software package ANSYS FLUENT [34]. Pressure-velocity coupling is accomplished through the PISO algorithm with non-iterative time advancement, except for the first transient time step, when multiple iterations are necessary to ensure that the absolute velocity field is replaced by the relative velocity field. We note that our phase change model is compatible with non-iterative time advancement schemes (ANSYS Fluent 15) because the interface is explicitly set to the saturation temperature at each iteration. The Green-Gauss node-based method is employed for accurately calculating scalar gradients and the Geo-Reconstruct method for volume-fraction discretization keeps the interface sharply resolved. The PRESTO and Third-Order Upwind (MUSCL) schemes are employed for discretization of pressure and momentum, respectively. A variable time step is set to maintain global Courant number equal to 0.05 in the simulation. The typical residuals in mass, momentum, and energy are

on the order of $10^{-12}, 10^{-7}$, and $10^{-12}$, respectively, for the two-dimensional simulation and $10^{-12}, 10^{-6}$, and $10^{-12}$ for the three-dimensional simulation.

\subsection{Simulation Domains and Mesh Setup}

The two-dimensional axisymmetric simulation domain has a channel length $L$ that is 22 times the channel diameter $D$. The mesh is shown in Fig. 2(b). The cell length along the flow direction is uniformly $0.016 D$. In the radial direction, the mesh is divided into three regions. In regions around the axis of the channel $(0 D$ to $0.32 D$ from the axis), the radial extent of the cell is uniform $(0.016 D)$ and forms square mesh cells. In the intermediate region between the axis and the channel wall ( $0.32 D$ to $0.45 D$ from the axis, 15 cells), the mesh is gradually refined in the radial direction approaching the wall. At the region adjacent to the wall $(0.45 \mathrm{D}$ to $0.5 \mathrm{D}$ from the axis), the mesh is uniformly divided into 15 cells with a mesh size of $D / 300$. The mesh size in the liquid film region satisfies the recommendation of Magnini et al. [14] that more than 7 cells should exist in this region. A total of 68,750 rectangular cells were included in the simulation domain, with a maximum aspect ratio of 4.8 . 
The numerical treatment for spurious current suppression described in Pan et al. [19] and employed in this work allows for this larger aspect ratio to be used. Maintaining the cells square throughout the domain would have increased the overall mesh size by a factor of $\sim 13$ relative to the present rectilinear mesh.

The three-dimensional simulation domain has a channel length $L$ that is 16 times the square channel side dimension $W$. The mesh is shown in Fig. 2(c). The cell length along the flow direction ( $z$ direction) is uniformly $0.024 \mathrm{~W}$. In the $x$ and $y$ directions, the mesh is divided into three regions. In regions around the center of the channel $(0 \mathrm{~W}$ to $0.36 \mathrm{~W}$ from the respective plane of symmetry), the size of the cell is uniform $(0.024 \mathrm{~W})$ and

forms cubic mesh cells. In the intermediate region between the plane of symmetry and the channel wall $(0.36 \mathrm{~W}$ to $0.44 \mathrm{~W}$ from the plane of symmetry, 6 cells), the mesh is gradually refined from the center toward the wall. At the region adjacent to the wall ( $0.44 \mathrm{~W}$ to $0.5 \mathrm{~W}$ from the plane of symmetry), the mesh is uniformly divided into 10 cells. In this way, the mesh in the liquid film region also satisfies size recommendations. A total of 634,260 cuboidal cells were included in the simulation domain, with a maximum aspect ratio of 5.7.

\subsection{Implementation of the Moving-Reference-Frame Method}

A moving reference frame is employed to suppress spurious currents by artificially increasing the local velocity near the interface during the numerical solution (as demonstrated by [19]). The effectiveness of the suppression approach allows the use of non-uniform mesh elements that help to significantly reduce the numerical cost. While this method had been successfully employed for studying two-phase convective heat transfer without evaporation [35][36], several modifications are needed to adapt the technique for use in flow boiling simulations (as discussed in detail in Pan et al. [37]). In the present study, the moving reference frame is set to move in the flow direction, as shown in Fig. 2 (b and c). In this reference frame, the simulation domain, channel wall, and boundary conditions move upstream at the moving-reference-frame velocity

In the two-dimensional flow boiling case, because the location of the inlet is moving (due to the moving reference frame employed) into a region where the local velocity profile is not fully developed, the inlet velocity profile that is imposed as a boundary condition must evolve with simulation time. At time zero, the inlet velocity is uniform $\left(U_{0, \text { inlet }}=0.4 \mathrm{~m} / \mathrm{s}\right)$. Then, the inlet moves forward a distance of $U_{\text {ref }} \Delta t$ with the reference frame after each time step. The velocity profile on the inlet boundary at time step $n+1$ can be calculated per Pan et al. [37] as

$$
U_{a x, n+1}=\psi_{u}\left[\frac{\left(U_{c e l l, a x, n}-U_{a x, n}\right) U_{a x, n} \Delta t_{n}}{\Delta x_{a x}}+U_{a x, n}\right]
$$




$$
U_{r a, n+1}=\psi_{u}\left[\frac{\left(U_{c e l l, r a, n}-U_{y, n}\right) U_{r a, n} \Delta t_{n}}{\Delta x_{r a}}+U_{r a, n}\right]
$$

in which

$$
\psi_{u}=\frac{\int_{\text {inlet }}\left[\frac{\left(U_{c e l l, a x, n}-U_{x, n}\right) U_{x a x, n} \Delta t_{n}}{\Delta x_{a x}}+U_{a x, n}\right] d A}{U_{\text {in, inlet }} A_{\text {inlet }}}
$$

where the subscript cell indicates the center value of the cell adjacent to the inlet. The function $\Psi_{u}$ is employed to avoid the accumulation of small numerical errors in the velocity calculation between time steps. In the present simulation, the deviation of $\Psi_{u}$ from 1 is typically smaller than 0.002 .

For the three-dimensional case, the inlet velocity profile is fully developed (unlike the uniform velocity profile imposed for the 2D case to facilitate comparison with the literature), and does not need treatment with Eq. (12-14). The velocity of the moving reference frame $U_{\text {ref }}$ is set as $0.35 \mathrm{~m} / \mathrm{s}$ and $0.62 \mathrm{~m} / \mathrm{s}$ in the twodimensional and three-dimensional simulations, respectively, to ensure that the reference-frame velocity in the liquid film region is large enough to suppress any spurious currents, while at the same time retaining the heated region of the channel within the simulation domain.

\subsection{Implementation of the Phase Change Model}

In practice, the energy source term (Eq. (9)) is also applied on the vapor cells $\left(C_{v}=1\right)$ adjacent to the interfacial cells $\left(0<C_{v}<1\right)$ for numerical stability. In all the simulations conducted herein, even though Eq. (9) is applied to both the interfacial cells $\left(0<C_{v}<1\right)$ and vapor phase cells, $\left(C_{v}=0\right)$, most of the overall latent energy source ( $>99.9 \%$, as validated in Section 5.3 ) is added at the interface to balance the superheat transferred from the liquid phase. However, imposing Eq. (9) on the vapor phase mitigates the possible accumulation of source-term numerical errors, rendering the model more stable. The latent heat in Eq. (9) corresponds to a commensurate change in the liquid mass. While the liquid mass source should have a value opposite that of the vapor mass source, liquid mass cannot be added in the vapor phase directly; the small percentage of latent heat contributing to the liquid source term within the vapor phase is instead redistributed to the interface as

$$
S_{m, l}=\left(\psi_{s}+1\right) \frac{S_{h, l a}}{h_{f g}}, \quad \psi_{s}=\frac{\sum_{c_{i}=0} S_{m, l}}{\sum_{0<c_{l}<1} S_{m, l}}
$$

This above formulation evenly redistributes the net liquid mass source designated for vapor cells onto the interface. Since most of the latent energy source is automatically added on the interface, $\psi_{s}$ is usually smaller than 0.001 in the present simulations. 
Owing to the phase change model employed, and the reduction in overall number of cells enabled by the spurious current suppression technique, the numerical cost of the present model is very low. The simulation time on a lab computer (Intel Xeon Processor E3-1245 v3, Windows 7 64bit) for the results presented was $\sim 14 \mathrm{~h}$ without any parallelization for the two-dimensional problem and $\sim 16 \mathrm{~h}$ with 6 -core parallelization for the threedimensional problem.

\section{VALIDATION OF THE SATURATED-INTERFACE-VOLUME PHASE CHANGE MODEL}

The one-dimensional Stefan problem is used for validation of the proposed phase change model, as in previous studies [32][38][39]. Fig. 3 shows a layer of vapor between a superheated, isothermal wall and the surrounding saturated liquid. Heat transferred from the superheated wall temperature $T_{w}$ to the liquid-vapor interface causes evaporation of the liquid; the vapor layer thickness grows and the interface thus moves away from the wall. The analytical solution for the variation of interface position with time is given as:

$$
x(t)=2 \beta \sqrt{\frac{k_{v} t}{\rho_{v} c_{p, v}}}, \beta \exp \left(\beta^{2}\right) \operatorname{erf}(\beta)=\frac{c_{p, v}\left(T_{w}-T_{s a t}\right)}{h_{f g} \sqrt{\pi}}
$$

The one-dimensional Stefan problem is simulated with a two-dimensional mesh in the validation. The simulation domain is $1 \mathrm{~mm}$ long and $0.002 \mathrm{~mm}$ wide. A uniform square mesh with an edge length of $0.001 \mathrm{~mm}$ is used. The top and bottom of the domain are set as adiabatic walls that apply no shear force; the right side is treated as a uniform pressure outlet at a distance of $x=1 \mathrm{~mm}$ from the wall. At time zero, the cells adjacent to the wall have an initial vapor fraction of 0.5 and all other cells are filled with liquid. Similar to the flow boiling problem implementation (Section 4.3), the energy source term (Eq. (9)) is applied on both the interfacial cells (0 $\left.<C_{v}<1\right)$ and the liquid cells $\left(C_{v}=0\right)$ since the liquid phase is always physically saturated. This treatment avoids numerical instability for the extreme case where the interface is located exactly at the mesh face between the liquid and vapor phases.

The evaporation of three common coolants (R113, HFE7100, and water, with properties as shown in Table 1) is predicted at a wall superheat of $10 \mathrm{~K}$. A comparison of interface position obtained from the numerical simulation implementing the saturated-interface-volume phase change model (dots) and the analytical solution (lines) is shown in Fig. 4(a). Excellent agreement is obtained for all coolants; there is negligible deviation of the vapor layer thickness between the simulation and analytical solutions. The temperature distribution at $1 \mathrm{~s}$ is 
shown in Fig. 4(b). The temperature profile is linear in the vapor layer and the interface temperature is properly fixed at the saturation temperature.

\section{RESULTS AND DISCUSSION}

\subsection{D Axisymmetric Flow Boiling in a Circular Microchannel}

The vapor bubble interface motion and growth are shown superimposed on the temperature field in Fig. 5. It should be noted that in the moving-reference-frame simulation, the velocity and temperature profile at axial position $x_{r e f}$ and simulation time $t$ correspond to those at axial position $x_{0}=x_{r e f}+U_{r e f} t$ in a static reference frame. Starting from the initialized shape, the bubble interface geometry quickly assumes a realistic physics-driven profile within the adiabatic section of the microchannel (before $1.9 \mathrm{~ms}$ have elapsed). At $1.9 \mathrm{~ms}$, the liquid film thickness is $0.023 \mathrm{~mm}$ (at the location where the film becomes nearly uniform along a small portion of the bubble length just ahead of the thin neck region at the trailing edge), which agrees with numerical simulations under the same conditions $(0.0225 \mathrm{~mm}:[14])$ and the empirical correlation of Han and Shikazono [40] (0.02 $\mathrm{mm}$ ). At $2.51 \mathrm{~ms}$, the bubble nose just enters the heated portion of the channel. As the bubble travels further into the heated channel, the liquid-vapor interface touches the thermal boundary, spurring evaporation (for example, as shown at $5.59 \mathrm{~ms}$ in Fig. 5). As the liquid continues to evaporate, the vapor bubble grows with time. At $t=$ $10.49 \mathrm{~ms}$ ( $7.98 \mathrm{~ms}$ after the bubble nose enters into the heated portion of the channel), the bubble length is more than twice as large as its initial size. At this time, the liquid film thicknesses is $0.026 \mathrm{~mm}$, which also agrees with numerical results reported under the same conditions $(0.025 \mathrm{~mm}$ : [14]) and the empirical correlation $(0.027 \mathrm{~mm}$ : [41]).

The absolute location of the vapor bubble nose is quantitatively compared with simulation data from Magnini et al. [14] in Fig. 6(a). Since the bubble is not initialized in exactly the same location in both studies, the positions of the bubble nose are plotted with time from the instant that the bubble nose enters the heated portion of the channel (i.e., $x_{0}=8 D$ in the current simulations). The initial time in Fig. 6(a) corresponds to 4.52 $\mathrm{ms}$ in Magnini et al. [14] and $2.51 \mathrm{~ms}$ in the present simulation. Excellent agreement in the bubble nose position is observed. At a time $7.98 \mathrm{~ms}$ after the vapor bubble entered the heated portion in the present study, the bubble nose velocity is $1.072 \mathrm{~m} / \mathrm{s}$ while the bubble tail-end moves at $0.486 \mathrm{~m} / \mathrm{s}$. This agrees with results from Magnini et al. [14], who reported respective velocities at the comparable time of $1.07 \mathrm{~m} / \mathrm{s}$ and $0.47 \mathrm{~m} / \mathrm{s}$. The heat transfer 
coefficient along the wall, $7.98 \mathrm{~ms}$ after the bubble nose entered into the heated portion of the channel, is plotted in Fig. 6(b). Good quantitative agreement is again obtained with the predictions of Magnini et al. [14].

As a result of the cost-effective spurious current suppression method and saturated-interface-volume phase change model developed in the present study, similar numerical accuracy is demonstrated to have been achieved as compared with previous studies that generally must employ highly parallelized computational processing in order to predict the behavior of two-dimensional two-phase flows. As a rough comparison, these geometries can be simulated in a matter of hours using a single core of a high-performance desktop computer (versus days or weeks for the methods in the literature) owing to the low numerical cost, as demonstrated here. This computational efficiency of the methods developed highlights the potential for enabling accurate and tractable simulation of three-dimensional two-phase flows that contain thin-liquid-film flow structures, by leveraging parallel processing, as is explored in the next section.

\subsection{D Flow Boiling in a Square Microchannel}

The shape of the vapor bubble, temperature contours, and fluid flow fields are shown at two different time instants in Fig. 7 and Fig. 8. At a time of $1.7 \mathrm{~ms}$ (shown in Fig. 7), the interface of the bubble just touches the thermal boundary layer in the heated region. The bubble starts growing and the evaporation rate is small, as shown in Fig. 9, which plots the evaporation rate and bubble volume as a function of time. A recirculating flow field is present in front of the leading edge of the bubble; this is clearly observed in the streamlines on a reference frame fixed to the velocity bubble nose (Fig. 7(b)). In this reference frame, the liquid flows away from the bubble in the high-velocity central region of the channel and moves towards the bubble interface in the nearwall, low-velocity region. This recirculating flow also advects heat from the high-temperature, near-wall region toward the center of the channel and extends the thermal boundary layer over a larger portion of the vapor interface. The absolute flow field is shown in Fig. 7(c). No spurious currents are observed.

The bubble grows at an increasing rate as it travels further into the heated microchannel, as shown in Fig. 9. This is due to the increase in the local wall temperature and in the area of the evaporating interface. After $5.0 \mathrm{~ms}$ (the instant considered in Fig. 8), the bubble length has increased by $45.6 \%$ compared with the initial condition, while the volume has increased by $52.7 \%$. Because the temperature of the bubble surface is fixed at saturation, and the surface passes close to the channel wall being separated only by a thin liquid film, the bubble leaves behind a very thin thermal boundary layer (as shown in Fig. 8 (a and b)). As will be discussed later in this paper, this thinning of the boundary layer is shown to be a very important mechanism for cooling the heated wall. Due to intense evaporation from the enlarged thin liquid film area surrounding the bubble, the velocity of the bubble 
nose increases significantly, while the bubble tail velocity remains almost the same (Fig. 10). The absolute flow field is shown in Fig. 8 (c), and again, there are no unphysical spurious velocities.

The wall heat transfer coefficient and the corresponding bubble interface profile is shown in Fig. 11. The heat transfer coefficient of the single-phase flow is simulated for the same fluid and boundary conditions, but without introducing a vapor bubble into the channel; this allows an approximate evaluation of the contribution of the heat transfer due to phase change. The highest heat transfer enhancement relative to the single-phase case is located downstream of the bubble tail (rather than adjacent to the liquid thin film region). This observation can be attributed to the transient behavior of the liquid film between the evaporating interface and the heated wall, and is consistent with observations in the literature [14] for a circular microchannel. As the bubble moves through the channel and evaporates intensely at the location of the thinnest liquid film, the superheated wall experiences a lag time to cool down due to the sensible heat capacity of the liquid. A very thin thermal boundary layer is left trailing the bubble, which corresponds to the location of the highest heat transfer enhancement relative to the single-phase case.

The energy source associated with the simulated latent heat transfer is shown overlaid with the bubble interface in Fig. 12. Even though the heat source (Eq. 9) is applied to both the interface and the vapor phase to aid numerical stability, most of the energy sink is indeed concentrated on the interface as described in Section 4.3. The magnitude of the energy source around the cross-section of the bubble largely depends on the thickness of the liquid film, where a thinner film results in a higher local evaporative heat sink. The energy source magnitude is higher close to the center of the confining walls where the interface is thinner, while it is weaker near the corners where the liquid film is much thicker. Along the streamwise direction of the bubble profile, the evaporative heat sink increases near the trailing edge.

\section{CONCLUSIONS}

In this paper, a volume of fluid-based flow boiling model is proposed with computational expense-saving features that enable accurate simulation of two-phase flow and heat transfer at low numerical cost. In order to predict phase change at the liquid-vapor interface, heat and mass source terms are calculated using a novel, saturated-interface-volume phase change model; the interface cells are fixed at the saturation temperature for each iteration. Numerical oscillation of the source terms is thus eliminated, reducing numerical cost. The phase

change model is validated against the analytical solution for a one-dimensional Stefan problem. A moving- 
reference-frame method is employed to reduce the influence of numerical errors resulting from calculation of the surface tension force, and thus suppresses the development of spurious currents. This allows use of nonuniform meshes that can efficiently resolve high-aspect-ratio geometries and flow features, and significantly reduces the overall numerical expense relative to uniformly dense square meshes. The proposed model is used to simulate the growth of a vapor bubble in a heated two-dimensional axisymmetric microchannel. The bubble motion, liquid film thickness, bubble growth rate, and local heat transfer coefficient along the wall are quantitatively compared against an identical case in the literature; excellent quantitative agreement is achieved using the low-cost implementation approach developed here. Three-dimensional flow boiling in a microchannel is then investigated to demonstrate the cost-effectiveness of the present model and highlight the transport mechanisms it can reveal in more complex domains.

\section{Acknowledgements}

This material is based upon work supported by the Defense Advanced Research Projects Agency (DARPA) Microsystems Technology Office's (MTO) Intrachip/Interchip Enhanced Cooling (ICECool) Fundamentals program under Cooperative Agreement No. HR0011-13-2-0010. The authors would like to thank Dr. Avram Bar-Cohen, Program Manager, and his technical team at DARPA for their guidance and feedback on the work.

The content of the information does not necessarily reflect the position or the policy of the Government, and no official endorsement should be inferred. Distribution Statement A - Approved for public release; distribution unlimited.

\section{REFERENCES}

[1] M. Wörner, Numerical modeling of multiphase flows in microfluidics and micro process engineering: a review of methods and applications, Microfluidics and Nanofluidics 12 (2012) 841-886.

[2] S. Szczukiewicz, M. Magnini, J.R. Thome, Proposed model, ongoing experiments and latest numerical simulations of microchannel two-phase flow boiling, International Journal of Multiphase Flow 29 (2014) 84-101.

[3] T. Qin, Z. Tukovic, R.O. Grigoriev, Buoyancy-thermocapillary convection of volatile fluids under atmospheric conditions, International Journal of Heat and Mass Transfer 75 (2014) 284-301. 
[4] K. Yang, F. Hong, P. Cheng, A fully coupled numerical simulation of sessile droplet evaporation using Arbitrary Lagrangian-Eulerian formulation, International Journal of Heat and Mass Transfer 70 (2014) 409-420.

[5] L. Chen, S.V. Garimella, J.A. Reizes, E. Leonardi, Motion of interacting gas bubbles in a viscous liquid including wall-effects and evaporation, Numerical Heat Transfer A 31 (1997) 629-654.

[6] L. Chen, S.V. Garimella, J.A. Reizes, E. Leonardi, The development of a bubble rising in a viscous liquid, Journal of Fluid Mechanics 387 (1999) 61- 96.

[7] A. Mukherjee, S.G. Kandlikar, Numerical simulation of growth of a vapor bubble during flow boiling of water in a microchannel, Microfluidics and Nanofluidics 1 (1005) 137-145.

[8] S. Gong, P. Cheng, Numerical investigation of saturated flow boiling in microchannels by the Lattice Boltzmann method, Numerical Heat Transfer A 65 (1014) 644-661.

[9] H. Wang, Z. Pan, S.V. Garimella, Numerical investigation of heat and mass transfer from an evaporating meniscus in a heated open groove, International Journal of Heat and Mass Transfer 54 (2011) 3015-3023.

[10]Z. Pan, H. Wang, Benard-Marangoni instability on evaporating menisci in capillary channels, International Journal of Heat and Mass Transfer 63 (2013) 239-248.

[11] M.H. Yuan, Y.H. Yang, T.S. Li, Z.H. Hu, Numerical simulation of film boiling on a sphere with a volume of fluid interface tracking method, International Journal of Heat and Mass Transfer 51 (2008) 1646-1657.

[12] R. Zhuan, W. Wang, Flow pattern of boiling in micro-channel by numerical simulation, International Journal of Heat and Mass Transfer 55 (2012) 1741-1753.

[13] R. Gupta, D.F. Fletcher, B.S. Haynes, On the CFD modelling of Taylor flow in microchannels, Chemical Engineering Science 64 (2009) 2941-2950.

[14] M. Magnini, B. Pulvirenti, J.R. Thome, Numerical investigation of hydrodynamics and heat transfer of elongated bubbles during flow boiling in microchannel, International Journal of Heat and Mass Transfer 59 (2013) 451-471.

[15] M. Magnini, B. Pulvirenti, J.R. Thome, Numerical investigation of the influence of leading and sequential bubbles on slug flow boiling within a microchannel, International Journal of Thermal Science 71 (2013) 36-52. 
[16] S. Popinet, An accurate adaptive solver for surface-tension-driven interfacial flows, Journal of Computational Physics 228 (2009) 5838-5866.

[17] M. Sussman, E.G. Puckett, A coupled Level Set and Volume-of-Fluid Method for computing 3D and axisymmetric incompressible two-phase flows, Journal of Computational Physics 162 (2000) 301-337.

[18]D.L. Sun, W.Q. Tao, A coupled volume-of-fluid and level set (VOSET) method for computing incompressible two-phase flows, International Journal of Heat and Mass Transfer 53 (2010) 645-655.

[19]Z. Pan, J.A. Weibel, S.V. Garimella, Spurious current suppression in VOF-CSF simulation of slug flow through small channels, Numerical Heat Transfer A 67 (2015) 1-12.

[20] W.H. Lee, A Pressure Iteration Scheme for Two-Phase Flow Modeling, Multiphase Transport Fundamentals, Reactor Safety, Applications, Hemisphere Publishing, Washington D.C, 1980.

[21]H.L. Wu, X.F. Peng, P. Ye, Y. Eric Gong, Simulation of refrigerant flow boiling in serpentine tubes, International Journal of Heat and Mass Transfer 50 (2007) 1186-1195.

[22] C. Fang, M. David, A. Rogacs, K. Goodson, Volume of fluid simulation of boiling two-phase flow in a vapor-venting microchannel, Frontiers in Heat and Mass Transfer 1 (2010) 013002.

[23] C. Gorlé, P. Parida, H. Lee, F. Houdhmand, M. Asheghi, K. Goodson, Validation study for VOF simulations of boiling in a microchannel, ASME InterPACK, 2015-48129, San Francisco, California, USA.

[24] A. Esmaeeli, G. Tryggvason, Computations of film boiling. Part I: numerical method, Int. J. Heat Mass Transfer, 472004 5451-5461.

[25] C. Kunkelmann, K. Ibrahem, N. Schweizer, S. Herbert, P. Stephan, T. Gambaryan-Roisman, The effect of three-phase contact line speed on local evaporative heat transfer: experimental and numerical investigations, International Journal of Heat and Mass Transfer 55 (2012) 1896-1904.

[26]H. Ganapathy, A. Shooshtari, K. Choo, S. Dessiatoun, M. Alshehhi, M. Ohadi, Volume of fluid-based numerical modeling of condensation heat transfer and fluid flow characteristics in microchannels, International Journal of Heat and Mass Transfer 65 (2013) 62-72.

[27] Y.Y. Tsui, S.W. Lin, Y.N. Lai, F.C. Wu, Phase change calculations for film boiling flows. International Journal of Heat and Mass Transfer 70 (2014) 745-757.

[28]D. Sun, J. Xu, Q. Chen, Modeling of the evaporation and condensation phase-change problems with FLUENT, Numerical Heat Transfer B 66 (2014) 326-342. 
[29]H. Wang, S.V. Garimella, J.Y. Murthy, Characteristics of an evaporating thin film in a microchannel, International Journal of Heat and Mass Transfer 50 (2007) 3933-3942.

[30] I. Tanasawa, Advances in condensation heat transfer, Advances in Heat Transfer, J.P. Hartnett, T.F. Irvine (Eds.), Academic Press, San Diego, 1991.

[31] C. Kunkelmann, P. Stephan, CFD simulation of boiling flows using the Volume-of-Fluid method within OpenFOAM, Numerical Heat Transfer A 56 (2009) 631-646.

[32] S. Hardt, F. Wondra, Evaporation model for interfacial flows based on a continuum-field representation of source terms, Journal of Computational Physics 227 (2008) 5871-5895.

[33] J.U. Brackbill, D.B. Kothe, C. Zemach, A continuum method for modeling surface tension, Journal of Computational Physics 100 (1992) 335-354.

[34] Fluent, ANSYS FLUENT 15.0: User's Guide, 2013, ANSYS-Fluent Inc., Canonsburg, PA.

[35] A.N. Asadolahi, R. Gupta, D.F. Fletcher, B.S. Haynes, CFD approaches for the simulation of hydrodynamics and heat transfer in Taylor flow, Chemical Engineering Science 66 (2011) 5575-5584.

[36] V. Talimi, Y.S. Muzychka, S. Kocabiyik, Numerical simulation of the pressure drop and heat transfer of two phase slug flows in microtubes using moving frame of reference technique, International Journal Heat and Mass Transfer 55 (2012) 6463-6472.

[37]Z. Pan, J.A. Weibel, S.V. Garimella, A cost-effective modeling approach for simulating phase change and flow boiling in microchannels, ASME InterPACK, 2015-48178, San Francisco, California, USA, 2015.

[38] G. Son, V.K. Dhir, Numerical simulation of film boiling near critical pressures with a level-set method, Journal of Heat Transfer 120 (1998) 183-192.

[39] S.W.J. Welch, J. Wilson, A volume of fluid based method for fluid flows with phase change, Journal of Computational Physics 160 (2000) 662-682.

[40] Y. Han, N. Shikazono, Measurement of the liquid film thickness in micro tube slug flow, International Journal of Heat and Fluid Flow 30 (2009) 842-853.

[41] Y. Han, N. Shikazono, The effect of bubble acceleration on the liquid film thickness in micro tubes, International Journal of Heat and Fluid Flow 31 (2010) 630-639. 


\section{TABLE CAPTIONS}

Table 1. Fluid properties at saturation temperature.

\section{FIGURE CAPTIONS}

Fig. 1 (a) Illustration of the saturated-interface-volume phase change model. (b) A flow chart of the saturated-interfacevolume phase change model implementation.

Fig. 2 Schematic illustration of: (a) a vapor bubble traveling through a heated microchannel with an upstream adiabatic portion and downstream heated wall; the simulation domains, boundary conditions, and mesh setups, and reference frames are shown for the (b) two-dimensional and (c) three-dimensional flow boiling cases investigated.

Fig. 3 Schematic illustration of: (a) the one-dimensional Stefan problem, and (b) the simulation domain, boundary conditions, and mesh setup.

Fig. 4 (a) Validation of the numerical model (dots) against the analytical solution (lines) for prediction of the evaporating interface location for a one-dimensional Stefan problem, and (b) the numerical prediction of the temperature field at $1 \mathrm{~s}$ (the vertical dashes on the $\mathrm{x}$-axis show the locations of the interface).

Fig. 5 Vapor bubble interface profile and temperature field contours for the vapor bubble flowing though the circular heated microchannel at multiple different time instants.

Fig. 6 (a) Bubble nose location with time upon entering the heated portion of the channel; (b) heat transfer coefficient along the wall $7.98 \mathrm{~ms}$ after the bubble nose enters the heated portion of the channel.

Fig. 7 Vapor bubble characteristics at $1.7 \mathrm{~ms}$ : (a) 3D view of the vapor bubble interface and the temperature field; (b) temperature field and flow streamlines of the relative velocity on a reference frame moving at the velocity of the vapor nose; (c) absolute velocity field and corresponding streamlines. The width of the channel is artificially stretched by a factor of 1.5 for visualization in parts (b) and (c).

Fig. 8 Vapor bubble interface, temperature and flow field in the microchannel at $5.0 \mathrm{~ms}$ : (a) 3D view of the vapor bubble and the temperature field; (b) temperature and streamlines of the relative velocity of the vapor nose; (c) the absolute velocity field and corresponding streamlines. The width of the channel is elongated by a factor of 1.5 in parts (b) and (c).

Fig. 9 Transient bubble volume and overall evaporation rate for the three-dimensional flow boiling case; the time instants of $1.7 \mathrm{~ms}$ and $5.0 \mathrm{~ms}$ correspond to the contour plots shown in Fig.7 and Fig. 8, respectively.

Fig. 10 (a) The location of the bubble nose and tail, and (b) the velocity of the bubble nose and tail after evaporation begins.

Fig. 11 The heat transfer coefficient on the wall and the corresponding bubble location at $5.0 \mathrm{~ms}$.

Fig. 12 Energy sink on the interface due to latent heat at $5.0 \mathrm{~ms}$. 
Table 1. Fluid properties at saturation temperature.

\begin{tabular}{lllllcc}
\hline \multirow{2}{*}{ Fluid Properties } & \multicolumn{2}{l}{ R113 at 1 atm } & \multicolumn{2}{l}{ HFE7100 at 1 atm } & \multicolumn{2}{c}{ Water at 10 atm } \\
\cline { 2 - 7 } & Liquid & Vapor & Liquid & Vapor & Liquid & Vapor \\
\hline Density $\left(\mathrm{kg} / \mathrm{m}^{3}\right)$ & 1502 & 8 & 1425 & 5.15 & 886.5 & 5.21 \\
Thermal conductivity $(\mathrm{W} / \mathrm{m} \mathrm{K})$ & 0.0632 & 0.00962 & 0.0618 & 0.0103 & 0.673 & 0.0365 \\
Heat capacity $(\mathrm{J} / \mathrm{kg})$ & 943 & 695 & 1430 & 900 & 4406.8 & 2718.5 \\
Viscosity $(\mathrm{kg} / \mathrm{m} \mathrm{s})$ & $4.77 \times 10^{-4}$ & $1.04 \times 10^{-5}$ & $3.56 \times 10^{-4}$ & $1.11 \times 10^{-5}$ & $1.50 \times 10^{-4}$ & $1.50 \times 10^{-5}$ \\
Surface tension $(\mathrm{N} / \mathrm{m})$ & 0.0144 & 0.0144 & 0.0136 & 0.0136 & 0.0421 & 0.0421 \\
Latent heat $(\mathrm{kJ} / \mathrm{kg})$ & 143.54 & 143.54 & 117.8 & 117.8 & 2012.6 & 2012.6 \\
\hline
\end{tabular}




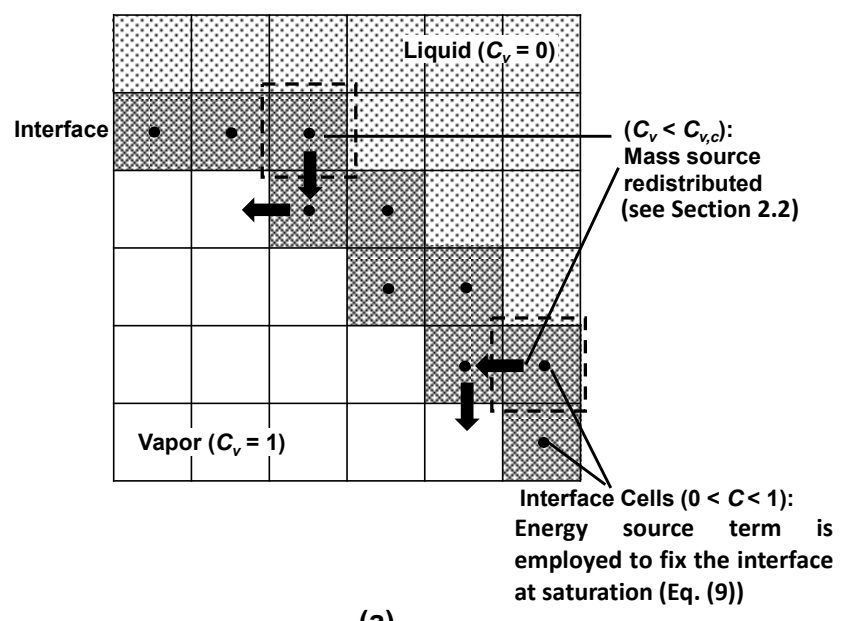

(a)

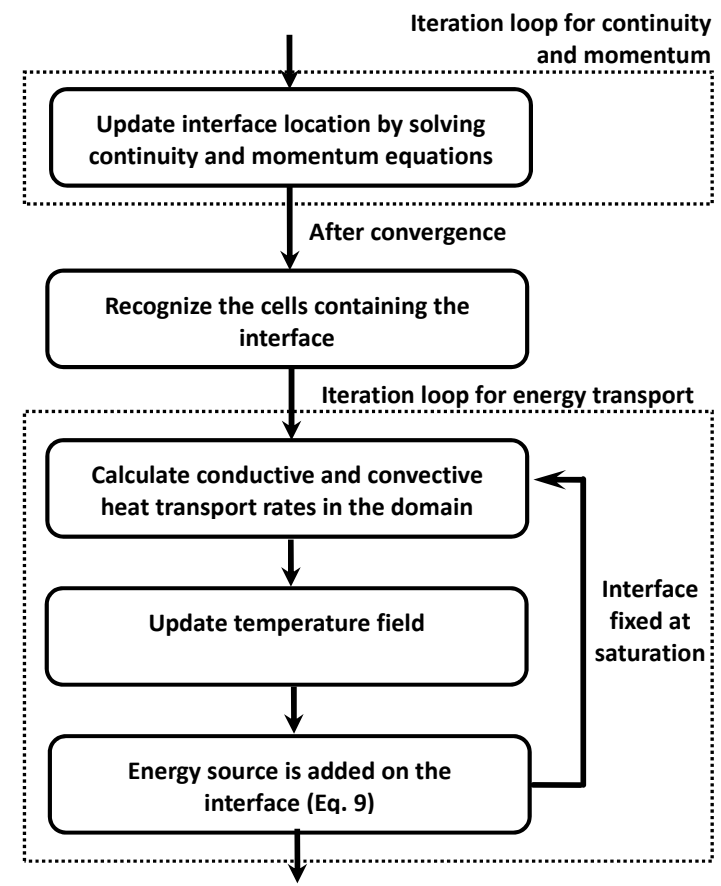

(b)

Fig. 1 (a) Illustration of the saturated-interface-volume phase change model. (b) A flow chart of the saturated-interface-volume phase change model implementation. 


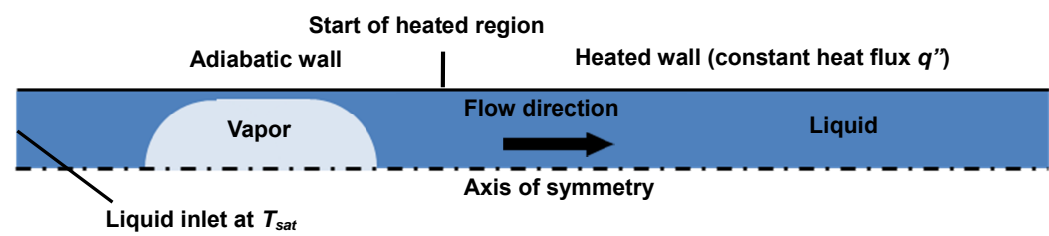

(a)

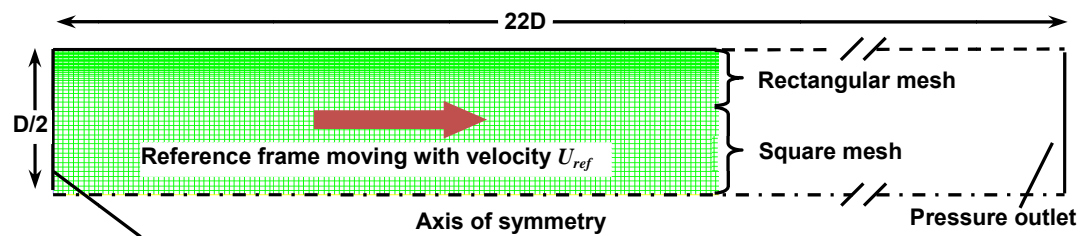

(b)

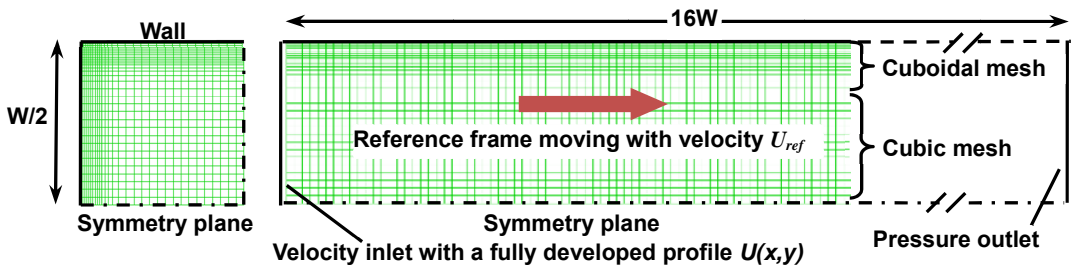

(c)

Fig. 2 Schematic illustration of: (a) a vapor bubble traveling through a heated microchannel with an upstream adiabatic portion and downstream heated wall; the simulation domains, boundary conditions, and mesh setups, and reference frames are shown for the (b) two-dimensional and (c) three-dimensional flow boiling cases investigated. 


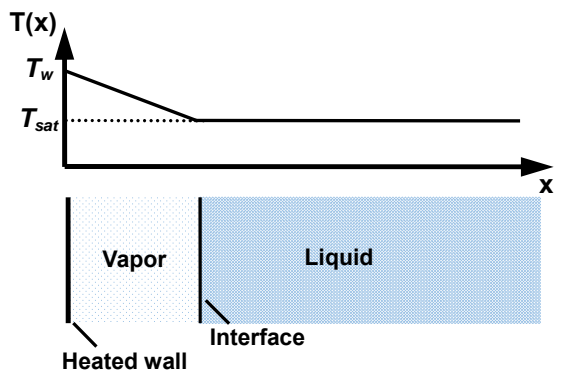

(a)

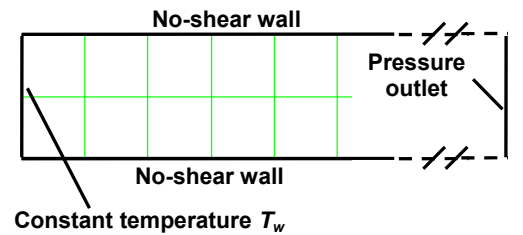

(b)

Fig. 3 Schematic illustration of: (a) the one-dimensional Stefan problem, and (b) the simulation domain, boundary conditions, and mesh setup. 


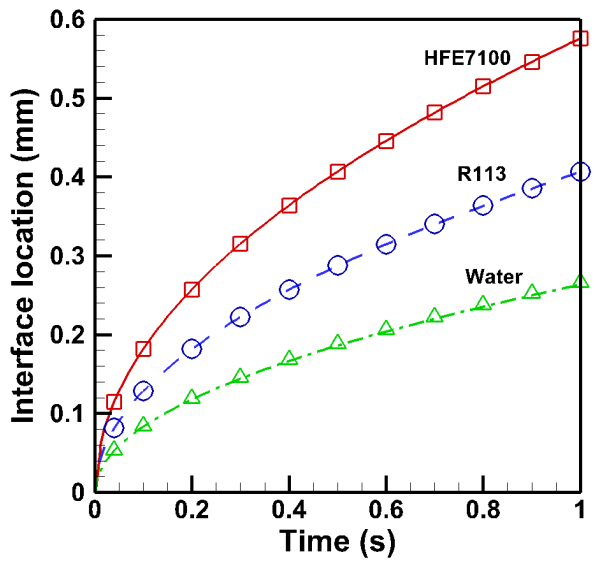

(a)

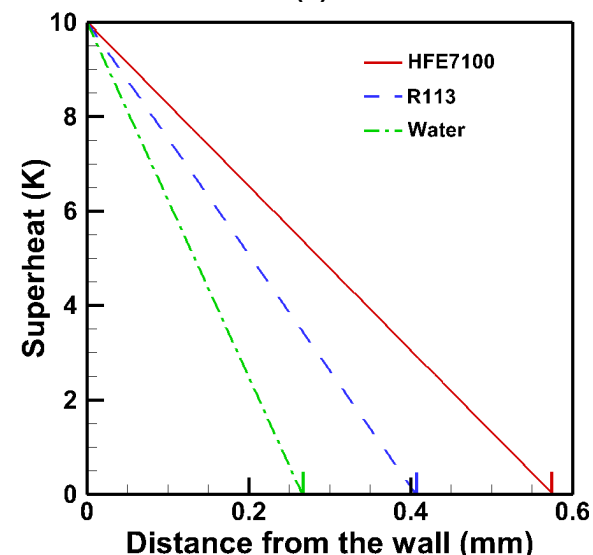

(b)

Fig. 4 (a) Validation of the numerical model (dots) against the analytical solution (lines) for prediction of the evaporating interface location for a one-dimensional Stefan problem, and (b) the numerical prediction of the temperature field at $1 \mathrm{~s}$ (the vertical dashes on the $x$-axis show the locations of the interface). 


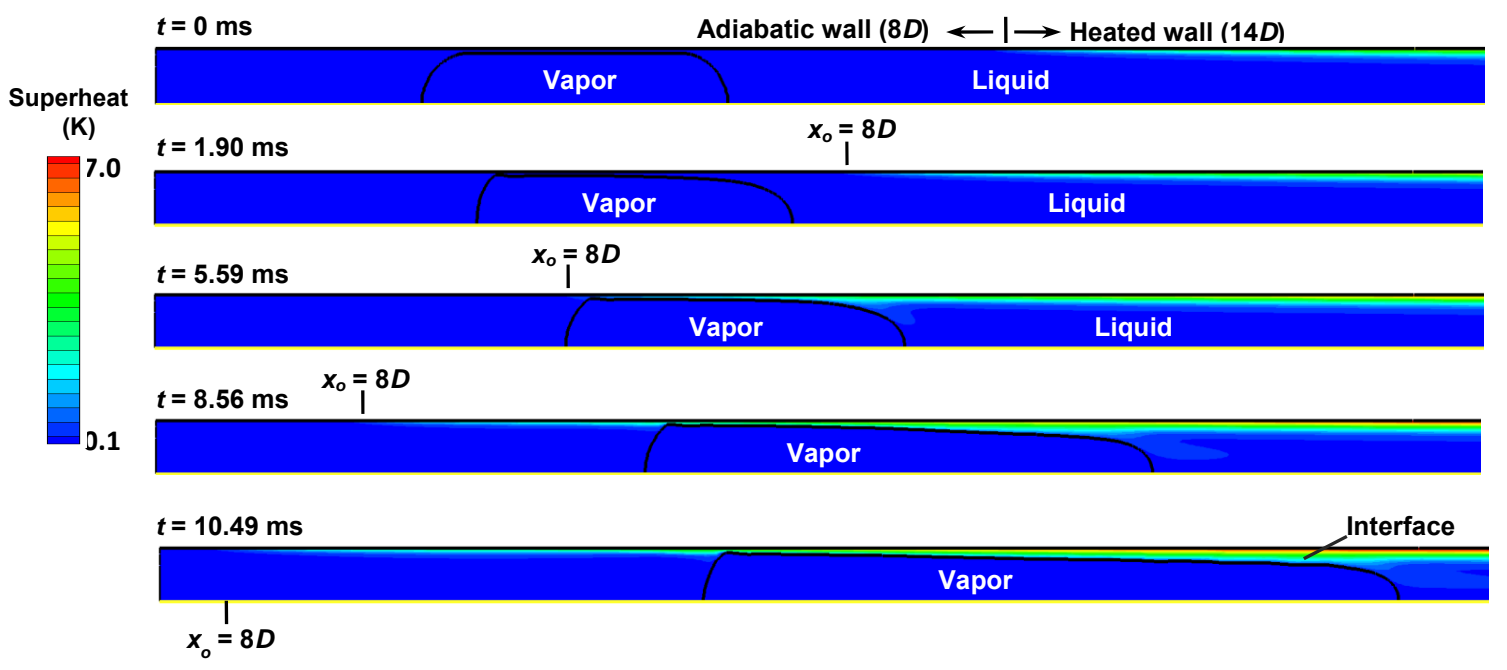

Fig. 5 Vapor bubble interface profile and temperature field contours for the vapor bubble flowing though the circular heated microchannel at multiple different time instants. 


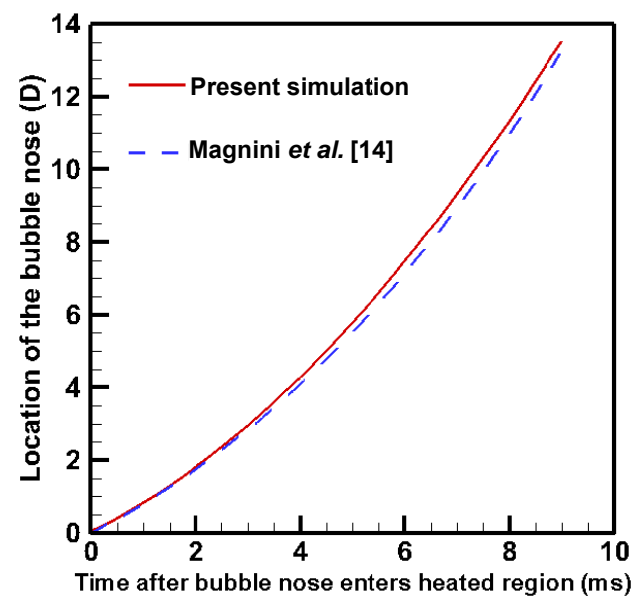

(a)

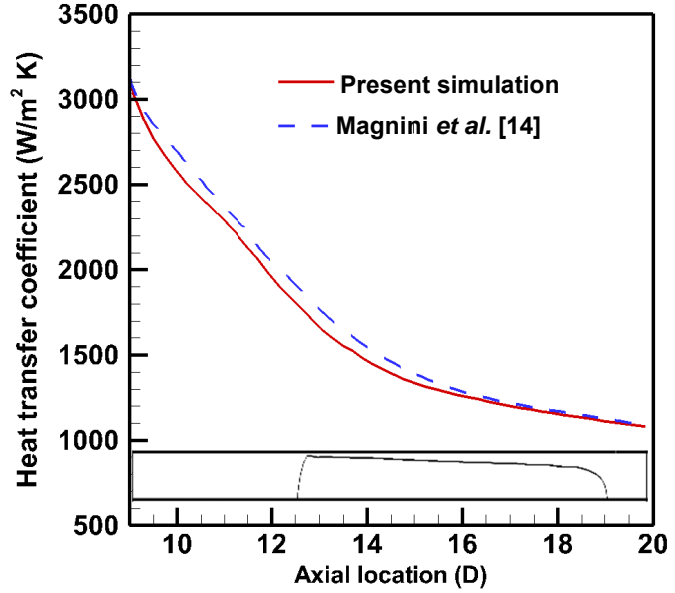

(b)

Fig. 6 (a) Bubble nose location with time upon entering the heated portion of the channel; (b) heat transfer coefficient along the wall $7.98 \mathrm{~ms}$ after the bubble nose enters the heated portion of the channel. 


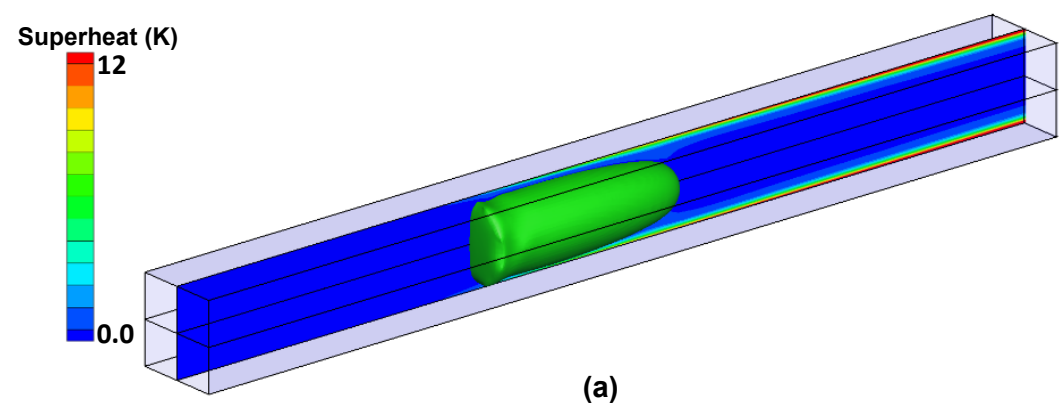

(a)

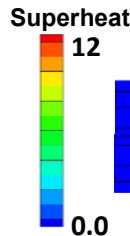

\section{eat (K)}

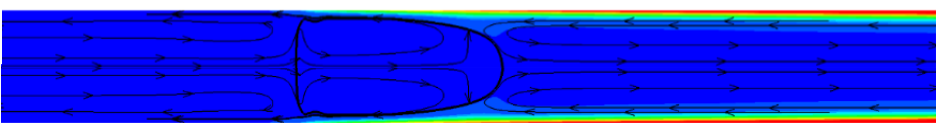

0.0

(b)

\section{Absolute velocity $(\mathrm{m} / \mathrm{s})$}

1.5
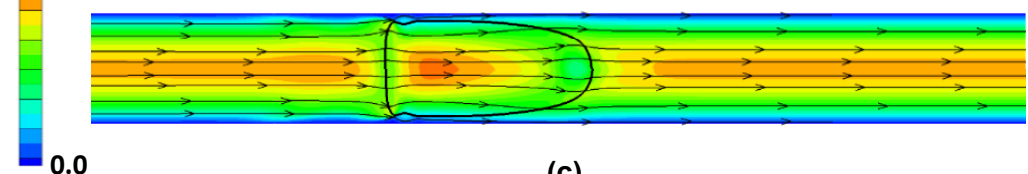

(c)

Fig. 7 Vapor bubble characteristics at $1.7 \mathrm{~ms}$ : (a) 3D view of the vapor bubble interface and the temperature field; (b) temperature field and flow streamlines of the relative velocity on a reference frame moving at the velocity of the vapor nose; (c) absolute velocity field and corresponding streamlines. The width of the channel is artificially stretched by a factor of 1.5 for visualization in parts (b) and (c). 


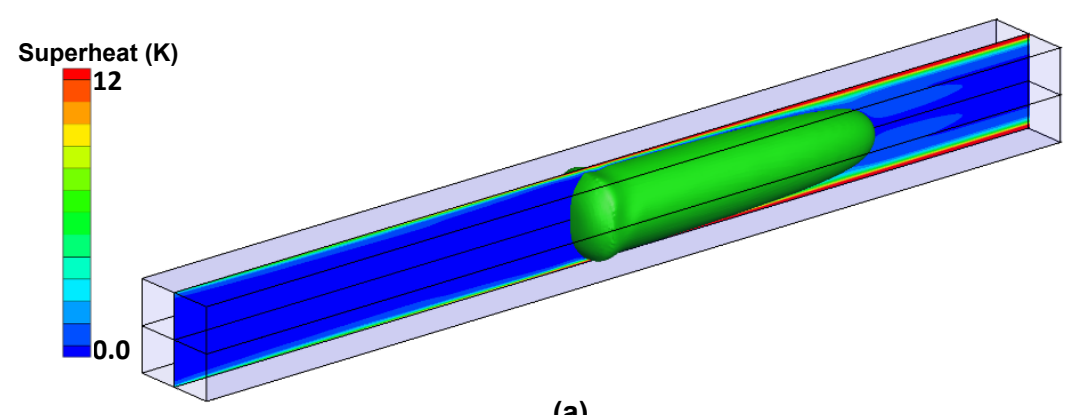

(a)
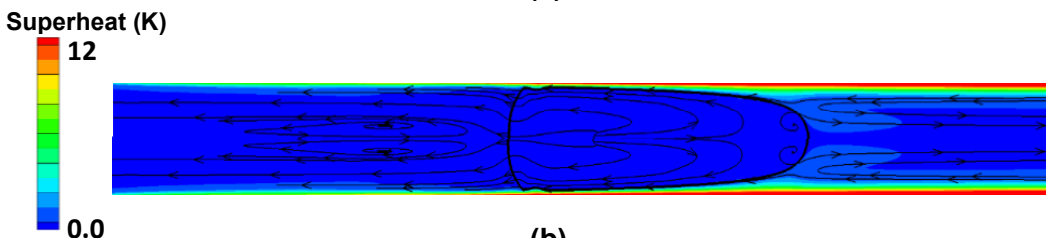

(b)

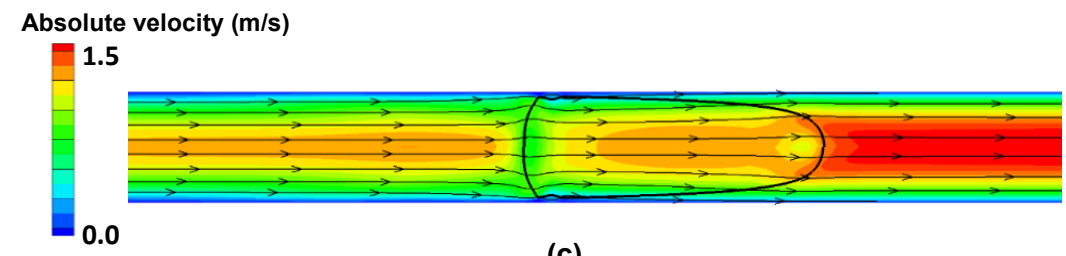

(c)

Fig. 8 Vapor bubble interface, temperature and flow field in the microchannel at $5.0 \mathrm{~ms}$ : (a) 3D view of the vapor bubble and the temperature field; (b) temperature and streamlines of the relative velocity of the vapor nose; (c) the absolute velocity field and corresponding streamlines. The width of the channel is elongated by a factor of 1.5 in parts (b) and (c). 


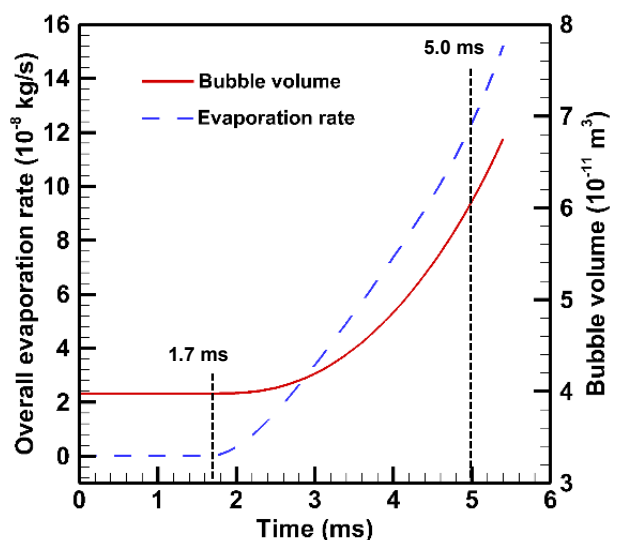

Fig. 9 Transient bubble volume and overall evaporation rate for the three-dimensional flow boiling case; the time instants of $1.7 \mathrm{~ms}$ and $5.0 \mathrm{~ms}$ correspond to the contour plots shown in Fig.7 and Fig. 8, respectively. 


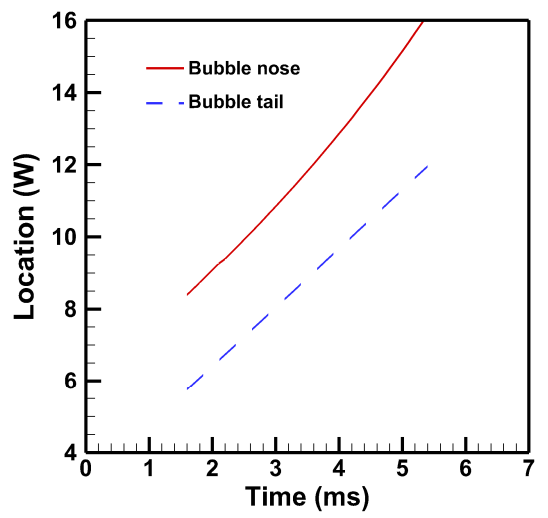

(a)

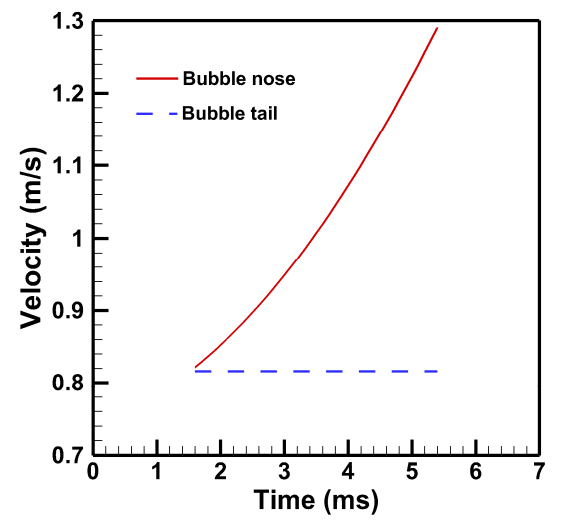

(b)

Fig. 10 (a) The location of the bubble nose and tail, and (b) the velocity of the bubble nose and tail after evaporation begins. 


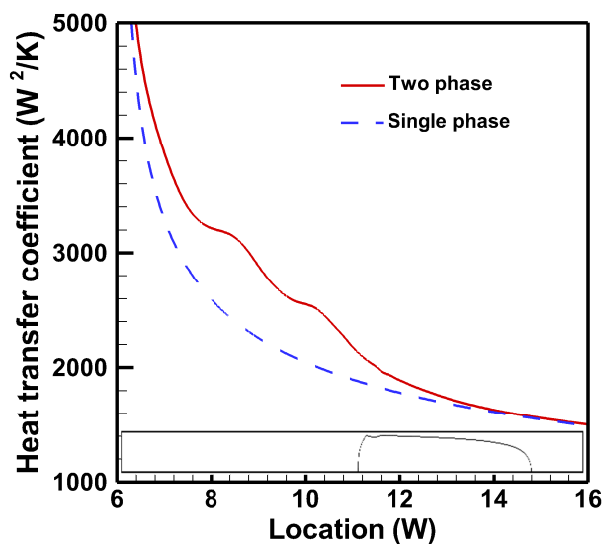

Fig. 11 The heat transfer coefficient on the wall and the corresponding bubble location at $5.0 \mathrm{~ms}$. 


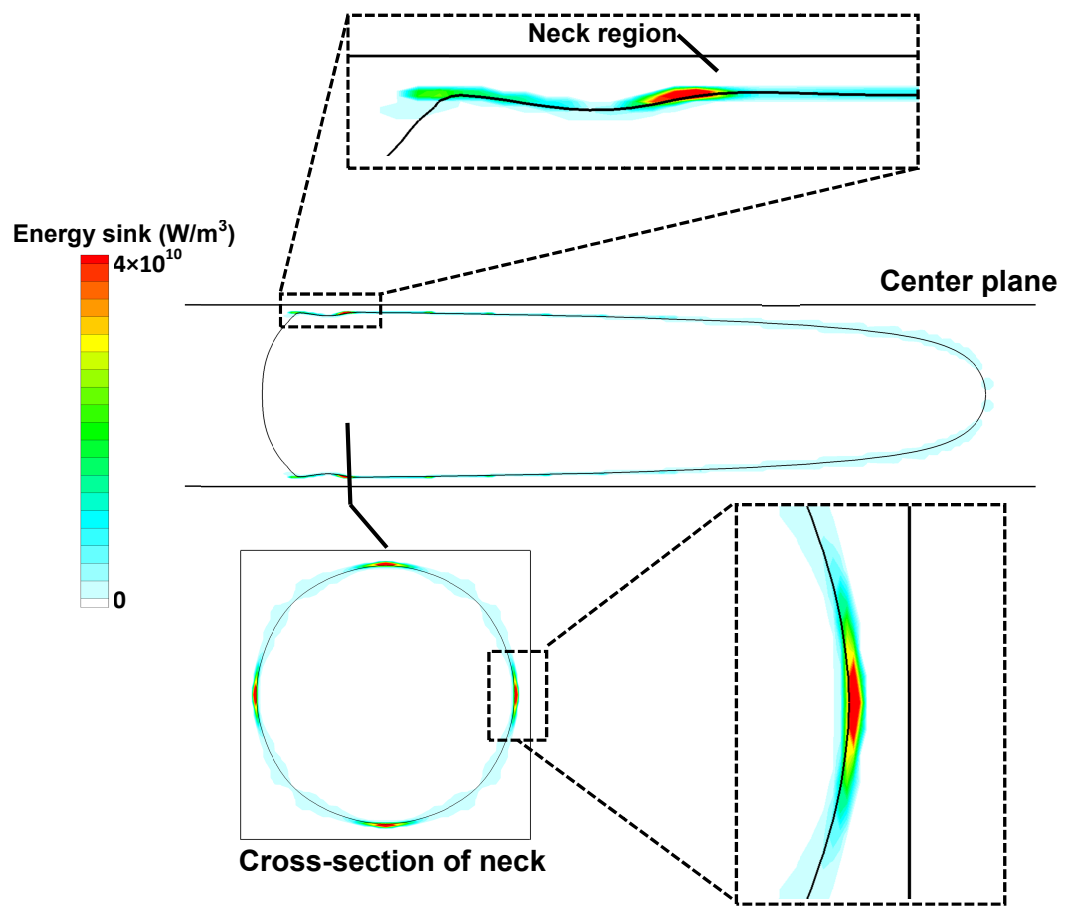

Fig. 12 Energy sink on the interface due to latent heat at $5.0 \mathrm{~ms}$. 